\title{
Infrared investigation of barite and gypsum crystallization: Evidence for an amorphous to crystalline transition
}

\author{
Franca Jones
}

\author{
Received (in $X X X, X X X)$ Xth $X X X X X X X X X 200 X$, Accepted Xth $X X X X X X X X X 200 X$ \\ ${ }_{5}$ First published on the web Xth $X X X X X X X X X 200 X$ \\ DOI: 10.1039/b000000x
}

Infrared was used to monitor the progression of barite $\left(\mathrm{BaSO}_{4}\right)$ and gypsum $\left(\mathrm{CaSO}_{4} \cdot 2 \mathrm{H}_{2} \mathrm{O}\right)$

crystallization. It was found that barite crystallized with broad infrared peaks that narrowed over

time. This was accentuated when an inhibitor was added. The infrared of gypsum in the early

10 stages showed a phase that was clearly different to either bassinite or gypsum. It is hypothesised

that in the absence of inhibitors, the rate-determining step for barite crystallization is the

transformation from an disordered solid to a crystalline solid as little water was observed to be

present during this process when inhibitors are not present. All of these findings are consistent

with a non-classical model of crystallization where disordered solids eventually rearrange to a

15 crystalline solid if not inhibited. It also suggests that water may be key to determining the

"lifetime" of the disordered clusters.

\section{Introduction}

When ions are dissolved in solvent it is a given that an 20 equilibrium will be established between hydrated ion pairs and hydrated ions. In this mechanism there are essentially only two interactions occurring; the ion-ion interaction and the ion-water interaction. However, why should single ion-pair complexes form and not larger ones? Solution clustering is not a new idea (see for 25 example 1,2 ). Concentration gradients, initially thought to conclusively show that solution clusters were formed were later shown not necessarily to be due to solution clustering alone. ${ }^{3}$ Recent experiments have been able to probe the solution and show that solution clustering occurs at least for organic 30 molecules ${ }^{4-9}$. The only inorganic mineral proven to show a clustering mechanism has been the calcium carbonate system ${ }^{10-12}$. Unfortunately, definitive data to prove or refute the existence of such clusters more generally has been elusive for low solubility inorganic mineral systems such as barium sulfate. This is due to 35 their low equilibrium solubilities, which in turn implies the solution concentrations of the clusters will be even lower still.

Unlike the difficulties with obtaining experimental data, molecular dynamics modelling is able to probe these systems and 40 determine many interesting properties. Modelling of barium sulfate $^{13,14}$ showed that the structure of barium sulfate stable in the simulation was significantly different to the bulk structure, if still crystalline. Other modelling evidence has shown that structuring of solution species ${ }^{15,16}$ is observed even for strong

45 electrolytes and that re-structuring of 'amorphous' clusters is a possible pathway to crystallization ${ }^{17}$. Thus, it was decided to investigate this area of research from a different perspective using vibrational spectroscopy. This manuscript presents the results of two crystallization systems; firstly, to see whether non50 classical nucleation was a more general phenomenon and secondly, to try and probe the role of water.
The non-classical crystallization mechanism hypothesis, as it is currently described, states that the combination of the 55 electrostatic ion-ion interactions and water interactions might be sufficient to stabilize these clusters in solution and while some argue that the inclusion of water reduces the entropy penalty expected if the clusters were completely solvent free ${ }^{12}$, others show it is of a lower free energy than free ions hydrated in ${ }_{60}$ solution ${ }^{18}$. Thus, depending on which argument one follows, the formation of these clusters may be either completely thermodynamically driven or only entropically driven. The interaction of the 'surface' ions with water might also stabilize any un-satisfied bonding (and/or charges) of the ions. Initially, ${ }_{65}$ the cluster is highly disordered and over time this becomes the crystalline particle via some mechanism. Thus, depending on the substrate, either removal of water or restructuring to form the crystalline structure may be rate determining. The hydroxyapatite system was one of the first to be observed to transform from a ${ }_{70}$ disordered to crystalline structure (see for example ${ }^{19,20}$ ) though definitive evidence for pre-nucleation clusters in this system is still missing.

This hypothetical mechanism has several advantages over the 75 current classical theory:

1. It can account for 'amorphous' solids precipitation by stabilization of the disordered (and/or hydrated) precursor phase.

2. It can account for crystal formation of much larger sizes than the critical nucleus size even at very high 80 supersaturations (where the particle size according to classical theory should be close to the critical nucleus size due to limited growth occurring).

3. It can account for hydrate and non-hydrate formation (kinetic/thermodynamic barrier to de-watering).

85

The work of Davey ${ }^{21}$ has shown that for urea, these two ratelimiting situations (desolvation versus restructuring) are also 
valid, thus demonstrating that this hypothesis is widely applicable (ie to both inorganic and organic species):

"However, while the coordination in solution may well be such as to satisfy all the local requirements of the crystal structure, the 5 transition to this latter state will obviously involve significant removal of water from the coordination environment of a central molecule."

Several predictions from this hypothesis can or already have 10 been tested:

a) water may be part of the structure prior to visible precipitation and the solids should be disordered at least on some time-scale ${ }^{18,22}$

b) clusters of ions of various ion numbers are present and 15 these sizes depend on the concentration of ions and solvent interactions ${ }^{10-12,23,24}$

c) the disordered structure can be stabilized and be precipitated in some circumstances (eg by impurities) ${ }^{11 \mathrm{a}, 25}$

20 In particular, the role of water has been seen to be important, particularly in stabilizing the disordered pre-nucleation cluster (see ref ${ }^{18}$ and references therein). It was determined that some of these predictions could also be tested on barite and gypsum by infrared since this is sensitive to changes in the water 25 environment (hydrogen bond vibrations are infrared active) and the infrared sulfate bands are relatively strong and well characterised. The comparison of barite to gypsum crystallization also allows us to probe the role of structural water. In addition, infrared bands can give some measure of crystallinity ${ }^{26-29}$. That

30 is, band positions can be sensitive to strain (see barite discussion below) and bandwidth can be related to the degree of disorder. ${ }^{26-}$ 29

\section{Experimental}

All materials were analytical grade reagents used as received. ${ }_{35}$ Ultrapure water (resistivity $>18 \mathrm{M} \Omega \mathrm{cm}$ ) was used in the preparation of all solutions.

Fourier transform infrared (FTIR, Brucker IFS66) using a horizontal attenuated total reflection (HATR) trough or planar 40 accessory with a ZnSe crystal was performed in the $4000-700$ or $1900-600 \mathrm{~cm}^{-1}$ region. The resolution was $4 \mathrm{~cm}^{-1}$ and 256 scans were taken for each run. The time taken to perform one 256 scan run was $\sim 2$ minutes. The cell was purged with dry nitrogen to reduce water vapour and a background spectrum was collected ${ }_{45}$ prior to $1 \mathrm{~mL}$ of solution being placed on the HATR crystal. Dry solids spectra were obtained on a Perkin-Elmer PE100 with a diamond ATR accessory using a $1900-600 \mathrm{~cm}^{-1}$ range at $4 \mathrm{~cm}^{-1}$ resolution and 8 scans were taken.

50 The solutions measured were ultrapure water, barium chloride solutions of different concentrations, sodium sulfate solutions of different concentrations, barium or calcium chloride mixed with sodium sulfate in a 1:1 ratio. In the case of the supersaturated solutions, 10 to $100 \mathrm{~mL}$ of the solution was prepared by dilutions 55 of stock solutions $(0.1 \mathrm{M})$ and $1 \mathrm{~mL}$ of this solution was then placed on the horizontal or in the trough ATR accessory. Spectra were then taken sequentially and the time stamp read directly from the file to calculate the elapsed time.

60 In the case of gypsum crystallization two experimental procedures were performed where, in the first, the solution (20 $\mathrm{mM}$ concentration of $\mathrm{Ca}^{2+}$ and $\mathrm{SO}_{4}{ }^{2-}$, SI index 0.05 for gypsum calculated using PHREEQC ${ }^{30}$ ) was simply left over time in the ATR trough accessory to crystallize. The second procedure ${ }_{65}$ involved gently blowing clean nitrogen gas over the surface of the solution (41 mM concentration of $\mathrm{Ca}^{2+}$ and $\mathrm{SO}_{4}{ }^{2-}$, SI index 0.40 for gypsum calculated using PHREEQC ${ }^{30}$ ) so as to increase the supersaturation by evaporation and accelerate crystallization. In this way, crystallization could be monitored over the time 70 range of initial crystallization through to the final gypsum product. For this reason we will refer to these experiments as either probing the "early crystallization stage" or the "later crystallization stages" respectively. The saturation index is defined as:

$75 \mathrm{SI}=\log (\mathrm{IAP} / \mathrm{Ksp})$

where $\mathrm{SI}=$ saturation index, $\mathrm{IAP}=$ ion activity product (product of the activities of all the ions) and $\mathrm{Ksp}=$ equilibrium solubility product.

In the case of barium sulfate crystallization barium chloride 80 solution was prepared at the desired concentration and sodium sulfate was added to the solution in a stoichiometric quantity before placing $1 \mathrm{~mL}$ of the solution on the horizontal plate ATR accessory or in the ATR trough accessory and the spectra collected over time. In the case where mellitic acid was present, 85 the final $\mathrm{Ba}^{2+}$ and $\mathrm{SO}_{4}{ }^{2-}$ concentration was $0.25 \mathrm{mM}$ and the mellitic acid was added to the barium chloride solution at $\mathrm{pH} 6$ to achieve a final concentration of $0.03 \mathrm{mM}$.

Details of the spectrum analysis to derive the spectra shown in 90 the figures are presented in the supplementary details. Essentially two methods were used:

i) Method 1: (note- this method was only used for barium sulfate crystallization.) Since the sulfate band was weak at the concentrations used for barium sulfate (see Figure 1 below), the barium+water spectrum and water vapour spectrum were subtracted (this method ignores ion-pair formation, sulfate hydration etc.)

ii) Method 2: The first spectrum was used as the reference spectrum so that all of the solvation of ions and ion100 pairing could be taken into consideration.

In all cases the subtraction between the data spectrum and the reference spectrum was limited to $(1 \pm 0.1) \mathrm{x}$ spectrum. Water vapour was then subtracted from the spectrum if this was significant. This allowed the crystallization behaviour to be 105 monitored over time.

The sulfate and water bands were followed during crystallization since the number and shape of the sulfate bands can also give qualitative information on the crystallinity of the 110 sulfate species (see below for detailed description of barite and gypsum infrared bands). 


\section{Barite (Pmna structure) $)^{31}$}

In solution (see Figure 1), the $T_{d}$ symmetry of the sulfate ion results in essentially one band (with the weak $980 \mathrm{~cm}^{-1}, v_{1}$ also sometimes observed) within this spectral region. As the 5 symmetry of the sulfate ion is decreased (in the formation of solids for example) the $v_{3}$ band splits. As can be seen (Figure 1) the sulfate ion in solution shows the strong band centred around $\sim 1100 \mathrm{~cm}^{-1}$. A water bend band is also observed with its maxima at $\sim 1645 \mathrm{~cm}^{-1}$ while for the dilute solution this band maxima 10 shifts to $\sim 1630 \mathrm{~cm}^{-1}$. Although water has been subtracted from this spectrum, the water bend region has a positive peak due to the hydration of sulfate (and possibly sodium) ions in solution.

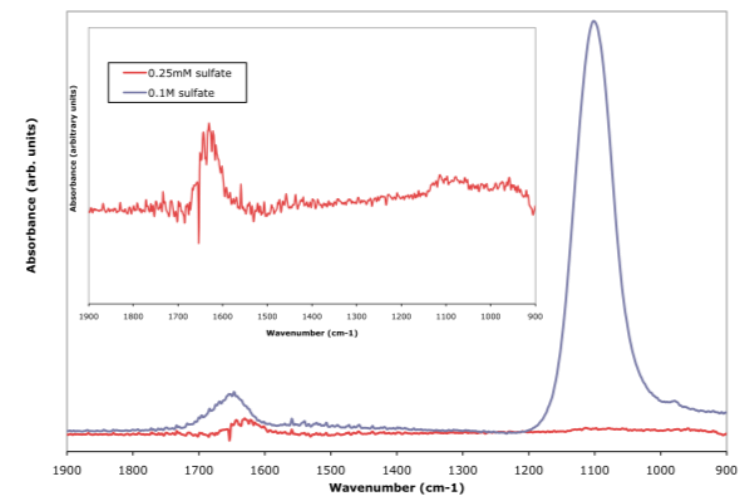

15 Figure 1. FTIR spectra of sodium sulfate at $0.1 \mathrm{M}$ (blue) and $0.25 \mathrm{mM}$ (red) which has been expanded in the insert

The barium chloride solution shows no spectral features other than the water bend, which, like the $0.25 \mathrm{mM}$ sulfate solution, has 20 its maximum centred around $1630 \mathrm{~cm}^{-1}$ (see supplementary information, SFigure 2). Once again, this positive water bend will be due to solvation of ions in solution. For pure crystalline barite (Figure 4), work by Wylde et al. ${ }^{32}$ and Adler \& $\operatorname{Kerr}^{33}$ have shown that, in the region $700-1900 \mathrm{~cm}^{-1}$, the following bands are 25 expected:

$\begin{array}{cc} & \text { Wavenumber }\left(\mathrm{cm}^{-1}\right) \\ & 980 \\ 30 & 1075 \\ & 1120 \\ & 1225\end{array}$

Vibrational mode $v_{1}$ $v_{3}$ $v_{3}$ $v_{3}$

The $1225 \mathrm{~cm}^{-1} v_{3}$ band (see Figure 4) is somewhat higher than found previously by Wylde et al. ${ }^{32}\left(\sim 1200 \mathrm{~cm}^{-1}\right)$ but is consistent 35 with Adler and $\mathrm{Kerr}^{33}$ and for barites formed by the mixed salt method. As the cation mass increases (in the group $\mathrm{Sr}, \mathrm{Ba}, \mathrm{Pb}$ ), the wavenumber of the $v_{3}$ band increases and this band is related to a decrease in symmetry of the structure and an increase in strain. $^{32,33}$

40

\section{Gypsum (A2/a structure) $)^{34}$}

For gypsum (Figure 2), the sulfate band is less well defined than for barite showing a broad band centred at approximately $1090 \mathrm{~cm}^{-1}$, the $\sim 1225 \mathrm{~cm}^{-1}$ and $1120 \mathrm{~cm}^{-1}$ bands are seen only as 45 very weak shoulders and the $980 \mathrm{~cm}^{-1}$ peak is closer to $1000 \mathrm{~cm}^{-1}$.
It cannot be directly compared to the barium sulfate spectrum because of the differences in their structure (barite being orthorhombic and gypsum being monoclinic). In addition, the water bend peaks are quite distinct as water sits in well-defined ${ }_{50}$ structural positions in the gypsum structure ${ }^{34}$ where one group of water molecules hydrogen bonds to the calcium ion via the water oxygen atom and the other water molecules hydrogen bond to the sulfate oxygen atom via the water hydrogen atom. These two peaks are assigned to the $A_{u}$ and $B_{u}$ modes of the $v_{2}$ water bend ${ }_{55}$ band $^{35-37}$ and have wavenumbers of 1690 and $1627 \mathrm{~cm}^{-1}$ respectively.

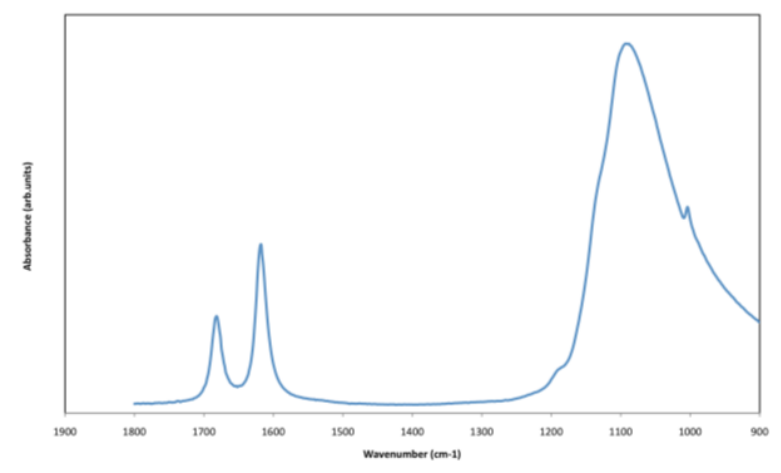

Figure 2. FTIR spectrum of solid gypsum in the $1900-900 \mathrm{~cm}^{-1}$ region

60

Gypsum also has a very well defined water stretch region for the same reason the water bend region is so well defined. At $2 \mathrm{~cm}^{-1}$ resolution, the $\mathrm{OH}$ stretch region is observed to have 3 peaks, though some have observed $4^{35-37}$. These peaks are ${ }_{65}$ assigned to the $A_{u}, B_{u}$ modes of the $v_{3}$ water stretch band ${ }^{35-37}$ having wavenumbers 3546 , and $3482 \mathrm{~cm}^{-1}$ and the $v_{1}$ of the $B_{u}$ mode having $3406 \mathrm{~cm}^{-1}$, these peaks being unresolved in the spectrum shown below (where the $B_{u}$ mode was found at the slightly lower wavenumber of $3401 \mathrm{~cm}^{-1}$, Figure 3). The $703245 \mathrm{~cm}^{-1}$ peak is a harmonic of the $v_{2}$ water bend.

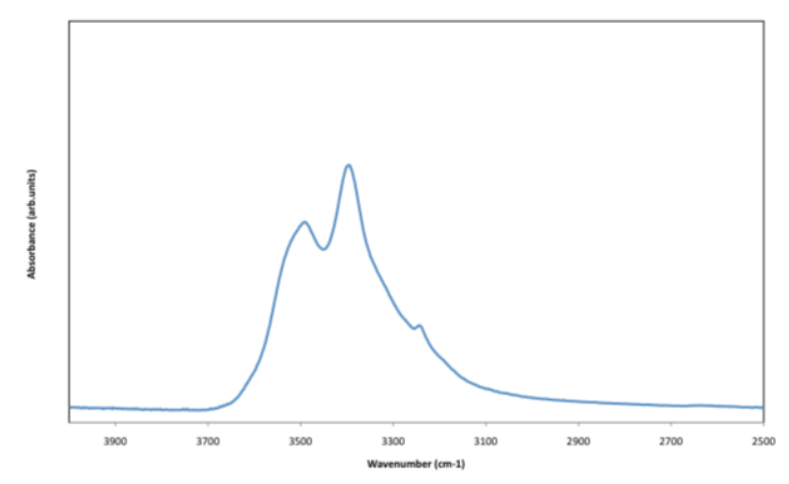

Figure 3. FTIR spectrum of solid gypsum in the $4000-2500 \mathrm{~cm}^{-1}$ region 


\section{Results and Discussion}

\section{Pure barium sulfate}

Firstly, barite crystallization at concentrations where homogenous crystallization $(\mathrm{SI}=64-125)$ is expected was 5 performed on an older, horizontal ATR accessory. In the homogenous nucleation regime it is expected that the particles will settle over time onto the ATR crystal and this will lead to an increase in the absorbance with time. It was found that within 2 minutes a crystalline sulfate spectrum is observed (See Figure 4); 10 the three $v_{3}$ bands at $1225 \mathrm{~cm}^{-1}, 1120 \mathrm{~cm}^{-1}$ and $1075 \mathrm{~cm}^{-1}$ are all observed as is the $v_{1}$ band at $980 \mathrm{~cm}^{-1}$. Thus, on the time-scale of the experiment, it was not possible to follow the crystallization process from solution to solids for these supersaturations on this ATR accessory (it is surmised that this older ATR accessory had 15 many scratches and led to faster crystallization).

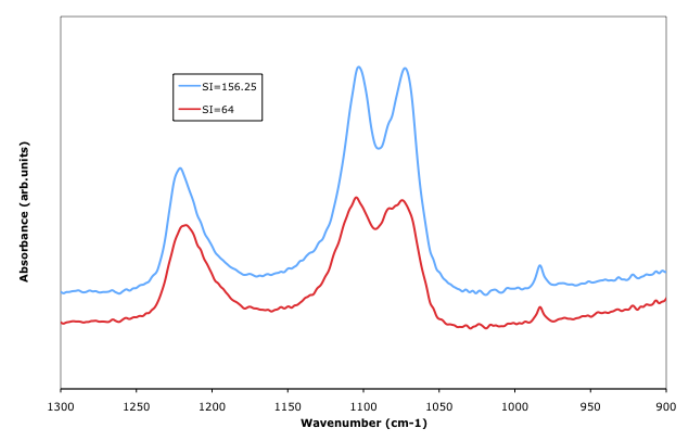

Figure 4. FTIR spectra of barite crystallized at different SI values

20

Below these values $(<\mathrm{SI}=49)$ however, the system is in the metastable zone. Thus, in this region, homogenous nucleation should not be observed. There remains however, the possibility that heterogenous nucleation onto the $\mathrm{ZnSe}$ crystal may occur. At

${ }_{25} \mathrm{SI}=9$ the spectra are seen to evolve over time as shown in Figure 5 due to heterogenous nucleation of the barium sulfate on the ATR crystal. In this supersaturation regime, the barium sulfate is expected to grow via the Birth and Spread mechanism albeit with a slow growth rate. As more nuclei or growth on the ATR crystal 30 occurs, the absorption will increase. In Firgure 5 the $v_{3}$ bands are weak and very broad suggesting a lack of crystallinity. Using Method 1 to analyse the spectra gives the results shown in Figure $5 \mathrm{a}$ and using Method 2 one obtains Figure 5b. Over a 20 minute time period in which the spectra were collected there is an 35 increase in band absorbance as well as a decrease in bandwidth (see Supplementary information, SFigure 3), where finally the three $v_{3}$ bands are observable. For SI $=9$ it takes $\sim 14$ minutes for the bands to develop to their maximum intensity (see Supplementary information SFigure 4). If the barite were 40 completely crystalline, the two bands centred around $1100 \mathrm{~cm}^{-1}$ should be resolvable since this peak is well above the noise level. This is observed only after 12 minutes has elapsed (using Method 1). This suggests that crystallinity is developing over time. This is more clearly seen when Method 2 is used to analyse the data. ${ }_{45}$ Here the water bend peak almost disappears and while bands above $1200 \mathrm{~cm}^{-1}$ and around $1100 \mathrm{~cm}^{-1}$ are observed they are broad and the $v_{3}$ bands are not all resolved at times $\leq 6$ minutes.

Thus, initially, less crystalline particles are formed that 50 become larger and more crystalline over time. This work was then repeated on a pristine ATR trough accessory in the homogenous nucleation regime. This newer accessory allowed for SI=100 to be monitored. It is found (Supplementary information, SFigure 4) that the $1120 \mathrm{~cm}^{-1}$ and the $1075 \mathrm{~cm}^{-1}$ 55 peaks are almost resolvable as soon as they emerge from the background. This suggests that, for barium sulfate, as supersaturation is increased the lifetime of any disordered solids decreases and the transition to a crystalline product is faster.

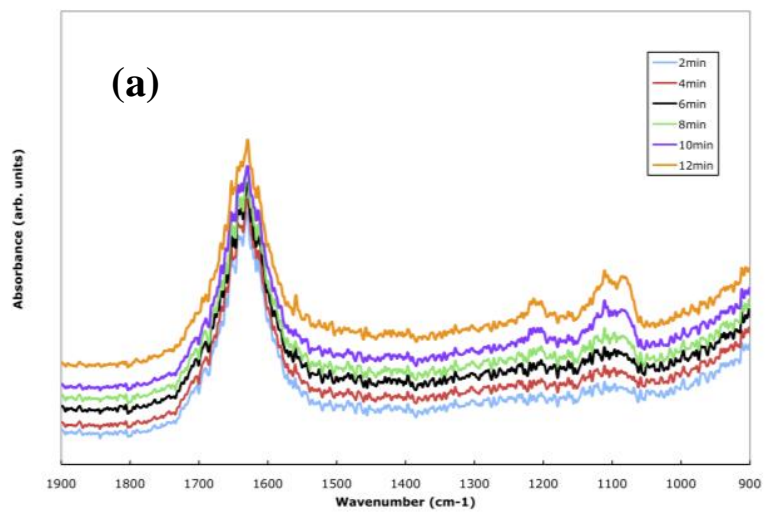

60

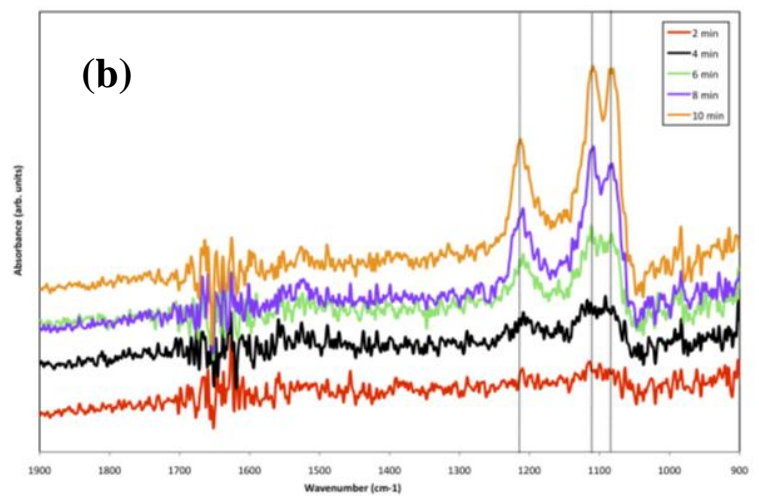

Figure 5. Infrared spectra of barium sulfate precipitated at $\mathrm{SI}=9$ at different times by (a) Method 1 and (b) Method 2 (spectra have been 65 offset in $y$-axis for clearer comparison)

A genuine issue can be raised about whether infrared band changes are purely related to changes in disorder or are particle size dependant. Literature shows that both particle size as well as 70 their degree of order/disorder influences the broadness of infrared bands $^{29,38}$, sometimes in opposing ways. Most recently, Poduska et al., ${ }^{29}$ has shown that decreasing particle size alone does not result in significant broadening and so broad peaks should relate to the extent of disorder only. Sensitivity of the infrared 75 instrument to species in solution or in solid form is only 
dependant on the signal to noise (which can be improved by counting for longer or having a more intense beam), however, for barite the low solubility of barium sulfate in solution may make this an issue.

Barium sulfate crystallization in the presence of mellitic acid

The interesting question then arises as to how barium sulfate might crystallize in the presence of inhibitors and what impact they have on the disordered to ordered solid transition. Mellitic 10 acid was chosen as the inhibitor for this work for two simple reasons:

i) it is a strong inhibitor and

ii) the carboxylate bands do not interfere with the sulfate bands in the infrared

15 spectrum

The presence of mellitic acid is known to form nanoparticles of barite that then self assemble over time to form mesocrystals. ${ }^{25} \mathrm{In}$ these experiments, although the SI is nominally equal to 625 , this does not take into consideration the complexation of barium ions 20 with mellitic acid. While this would normally be in the homogenous nucleation region, due to the mellitic acid being an inhibitor the system may or may not homogenously nucleate. In any event, the infrared peaks will grow in intensity as more and more solids deposit or grow on the ATR crystal.

When crystallizing barite from solutions containing mellitic acid the FTIR spectra were notably different from those without inhibitor present. When using Method 1 for the spectral analysis the formation of solids shows a sulfate band with (Figure 6a, 2

$30 \mathrm{~min})$ a $\mathrm{T}_{\mathrm{d}}$ sulfate symmetry, similar to sulfate in solution. At 15 minutes the first signs of a semicrystalline solid appears, as per $\mathrm{SI}=9,12$ minutes (Figure 5). Also at this time the mellitic acid bands begin to emerge at $\sim 1550,1350$ and $1410 \mathrm{~cm}^{-1}{ }^{39}$ The bands for sulfate are broader generally in the presence of mellitic 35 acid and the $1230 \mathrm{~cm}^{-1}$ band of sulfate has shifted to a much lower wavenumber, namely $1190 \mathrm{~cm}^{-1}$. This is also observed when greater strain and lower symmetry sulfate solids are formed. ${ }^{33}$ Thus, these results are consistent with a disordered structure slowly becoming a more crystalline solid. It is 40 interesting to note that at 2 minutes such a disordered sulfate band is observed. Solution sulfate can be ruled out since, in Figure 1, it is shown that at $0.25 \mathrm{mM}$ sulfate is barely observable, thus this is due to a highly disordered solid of barium sulfate in the presence of mellitic acid. Also there appears to be some water 45 loss during the crystallization period (see Supplementary information, SFigure 5).
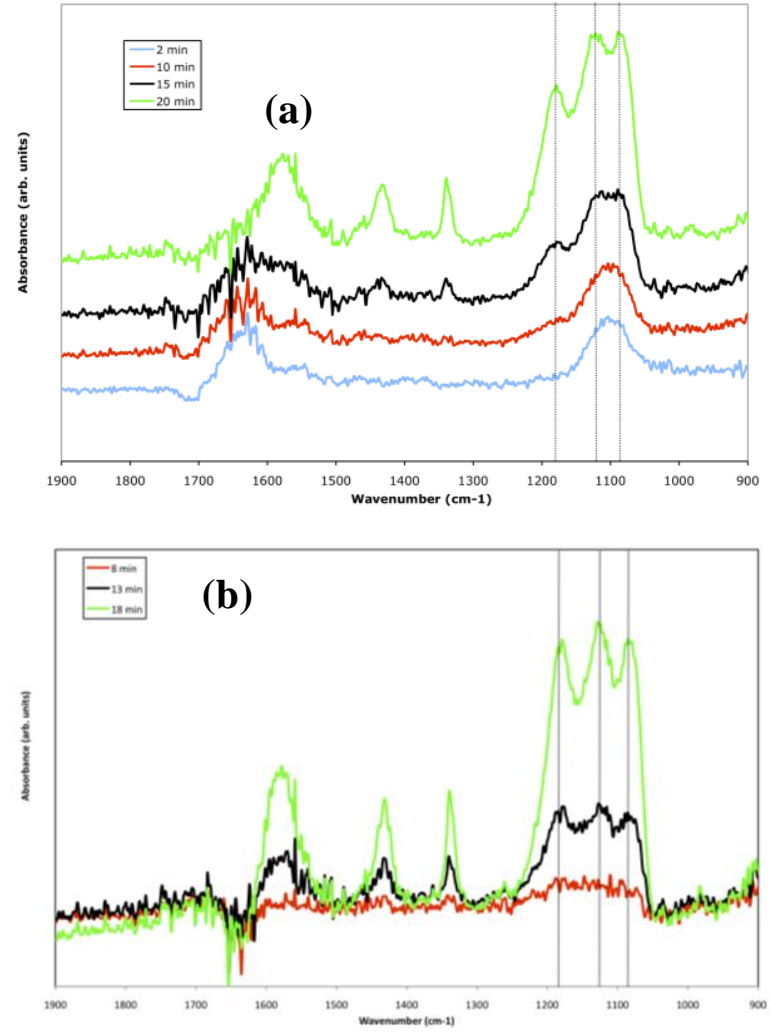

50 Figure 6. Infrared spectra of barium sulfate crystallization in the presence of mellitic acid at different times analysed by (a) Method 1 and (b) Method 2

Using Method 2 for the analysis similar effects are observed ${ }_{55}$ except that the water bend disappears and the emergence of the mellitic acid bands are more clearly visible (Figure 6b). The broadness of the sulfate band is also accentuated in this analysis and the crystalline solid still has the $1225 \mathrm{~cm}^{-1}$ peak shifted to lower wavenumbers suggesting a strained solid. Thus for this 60 inhibitor, despite the barium and sulfate concentration being much higher, the mellitic acid stabilises a disordered solid prior to formation of a crystalline one. Mellitic acid, as previously stated, is known to stabilize nanoparticles of barite prior to selfassembly. ${ }^{25}$ In classical nucleation, inhibition either occurs by 65 changing the surface free energy of the critical nuclei and/or by altering the growth rate of the growing nuclei. While there is no doubt that the mellitic acid is adsorbing onto the small particles (be they disordered or crystalline) and altering the surface free energy of those particles, the existence of a disordered solid tends 70 to support the non-classical view of crystallization.

Further support for a disordered barite particle rather than just a small barite particle was obtained from a TEM (transmission electron microscopy) investigation (Figure 7). A batch 75 crystallization experiment of barium sulfate in the presence of mellitic acid at $0.03 \mathrm{mM}$ as per $\operatorname{ref}^{25}$ was conducted with small TEM grids placed at the bottom of the vial. The TEM grids were then imaged and solids assessed by SAED (selected area electron diffraction) for crystallinity. 

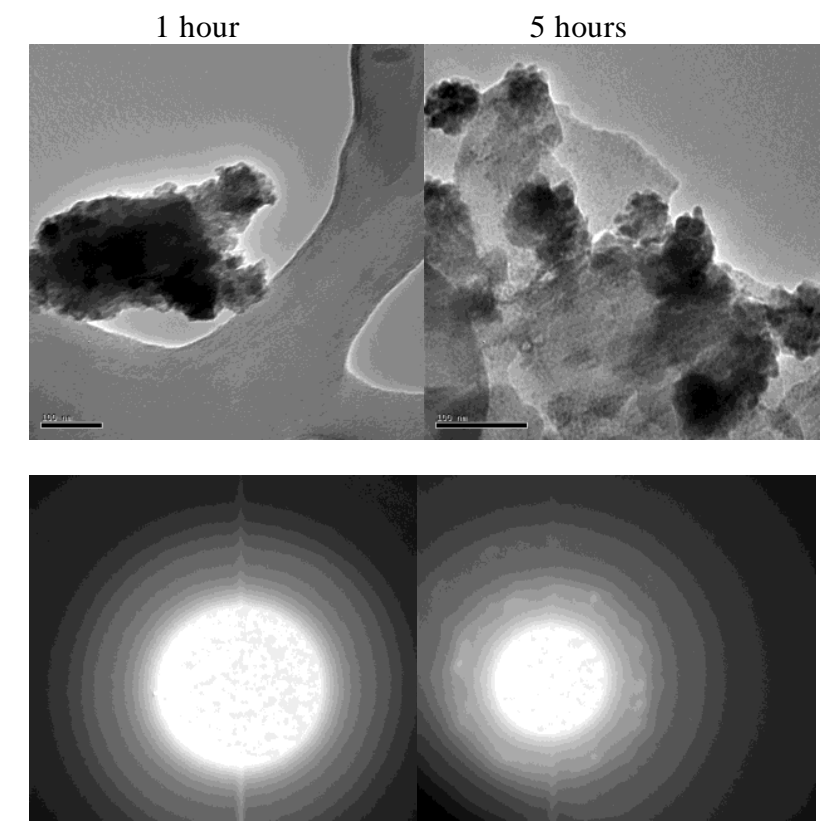

5 Figure 7. TEM and SAED images of barium sulfate particles formed in the presence of mellitic acid after 1 and 5 hours.

It can be seen that the particles between 1 and 5 hours are of the same order of magnitude in terms of size and yet spots 10 showing crystallinity are only observable in the 5 hour case. The FTIR, in that sense, is more sensitive than the TEM since FTIR shows crystallinity developing already at 15 minutes.

For barite then, infrared shows very little water present in the 15 disordered solids when inbitor is not present but does show a disordered to crystalline solid transition, at least in the presence of an inhibitor. However, the absence or presence of water may be obscured by the fast crystallization kinetics of barium sulfate. Future work is planned to conduct similar infrared experiments 20 using synchrotron radiation to probe more accurately the presence or absence of water in these structures.

\section{Gypsum}

Gypsum crystallization is quite different to barite in that the 25 changes observed over time are very slow in comparison to barite and only Method 2 was used to analyse the data. In addition, for both experimental conditions, heterogenous nucleation onto the ATR crystal rather than homogenous nucleation will occur. Thus, the solids are expected to grow on the ATR crystal and as this 30 occurs the infrared bands will grow in intensity. Looking at the "initial crystallization stages" (see 'Methods' section for an explanation of this term, Figure 8a) and the lower wavenumber region, little changes other than the sulfate and the water bend bands increase. In the "later stages of crystallization" caused by 35 the forced evaporation of the water, more structure develops in this region (Figure 9a). This is particularly true of the water bend band that slowly grows to show the two distinct water environments in the gypsum structure. In fact, the data suggests that the first water molecules to 'find' their place within the 40 structure are those having a vibrational band at $\sim 1640 \mathrm{~cm}^{-1}$.
Eventually these become the distinct $1690 \mathrm{~cm}^{-1}$ and $1627 \mathrm{~cm}^{-1}$ bands expected for gypsum. This is interesting as it suggests that the waters are in an average state to begin with but then split into two distinct groups, one having a higher vibrational energy and 45 one a lower.
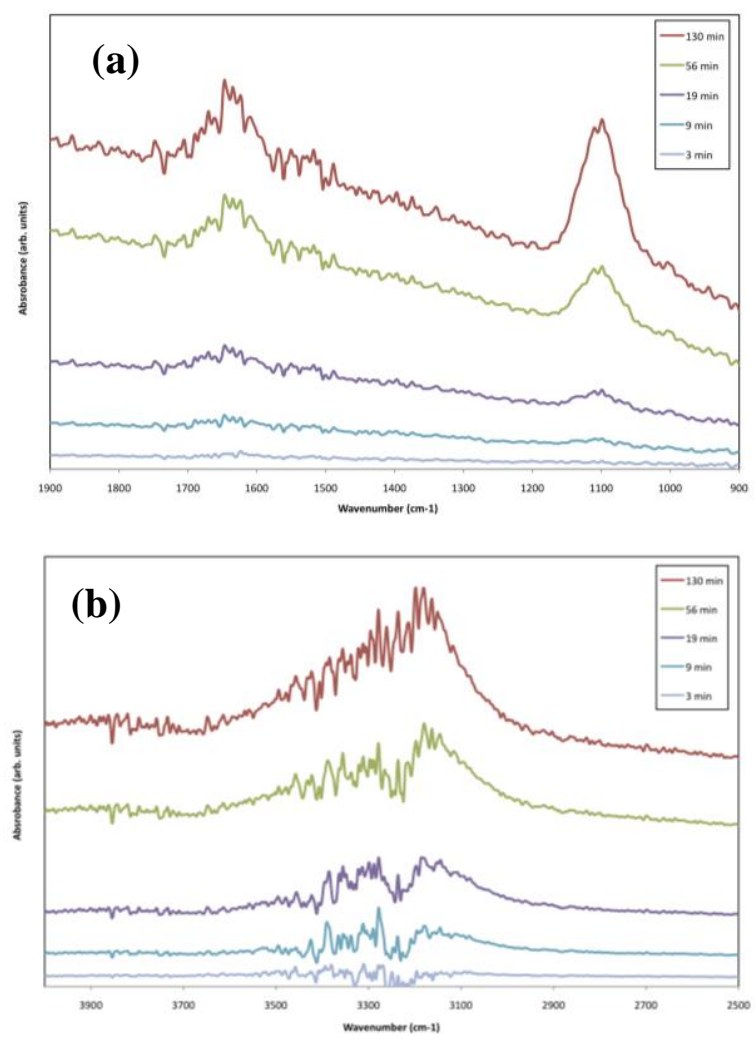

Figure 8. FTIR spectra of gypsum crystallization in the "early 50 stages" (a) 1900-900 and (b) 4000-2500 $\mathrm{cm}^{-1}$ region

In the water stretch region (Figure $8 b$ and $9 b$ ) there is also a clear transition from a broad feature to more well-defined bands, suggesting a disordered to crystalline transition. Initially, there is 55 a water stretch feature centred at $\sim 3200 \mathrm{~cm}^{-1}$, followed by the growth of a water stretch feature at $3400 \mathrm{~cm}^{-1}$. Only during the latter stages of crystallization does the water stretch band, at $3500 \mathrm{~cm}^{-1}$, become evident.

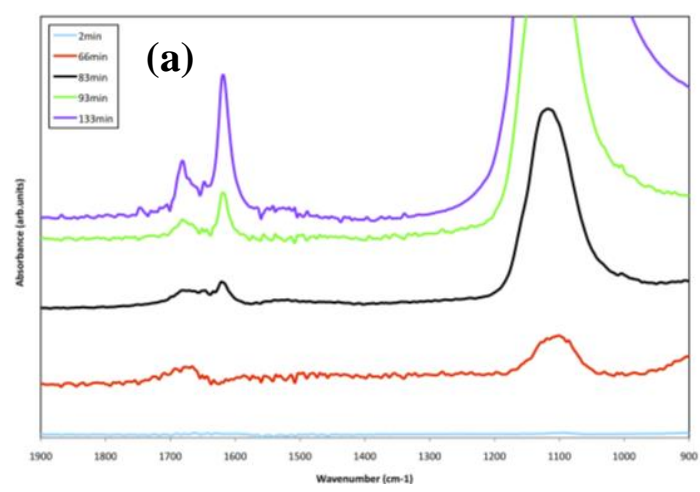




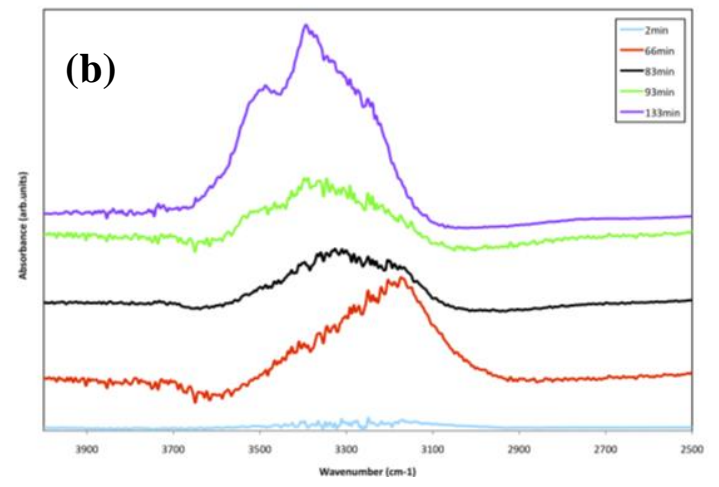

Figure 9. FTIR spectra of gypsum crystallization in the "latter stages" (a) 1900-900 and (b) $4000-2500 \mathrm{~cm}^{-1}$ region

5 This data suggests that initially, little water is present in the structure (at 3 minutes the flat nature of the spectrum for both $8 \mathrm{a}$ and $9 \mathrm{a}$ suggests the only water present is as pure water, within initial solution clusters or as waters of hydration around the ions since this is what is subtracted). As time continues, water is part 10 of the structure in a disordered fashion having a broad average vibrational energy of $1640 \mathrm{~cm}^{-1}$ and its harmonic is visible at $3200 \mathrm{~cm}^{-1}$. In the 'later stages' of crystallization this average water band diminishes as water begins to be in a more structured environment. In the water stretch region the $B_{u}$ mode is first seen 15 and the $A_{u}$ mode is finally resolvable only after long periods of crystallization. Thus, there is a clear trend from a more disordered to a more crystalline structure and that water is part of that process. An electron microscopic investigation of calcium sulfate has also recently seen the formation of 'amorphous' 20 nanoparticles $^{40}$ while another work showed bassanite (hemihydrate) was formed ${ }^{41}$. The hemihydrate can actually be excluded as forming in the early stages of this investigation. The hemihydrate has an FTIR spectrum similar to that of gypsum with bands at 3610,3555 and $1620 \mathrm{~cm}^{-1} \cdot 37,42$ None of these bands are 25 observed in the early stages of crystallization. In the latter stages of crystallization it is possible that both bassanite and gypsum exist, as found in ${ }^{41}$.

The changes observed in the infrared spectrum over time are 30 so significant that they cannot be explained by particle size effects. Having said this, surfaces can often contain different functionalities to the bulk due to their unique bonding arrangement. ${ }^{43} \mathrm{~A}$ more hydrated bassanite has been observed at higher humidities ${ }^{44}$, which could be a good candidate for any 35 proposed surface structure. However, given the penetration depth of the evanescent wave in the ATR method ${ }^{45}$, this should mean that both the surface and bulk bands should be observable. The water bend region is so broad that such a conclusion is plausible but in the water stretch region, bands that would be associated 40 with both surface and bulk structures are not observed (which would be seen at $>3400 \mathrm{~cm}^{-1}$ based on comparison to bassanite). Thus, this cannot explain the observed differences in the spectra when gypsum is crystallizing. The FTIR spectra suggest that initially, a disordered solid phase as found in $\mathrm{ref}^{40}$ is being 45 formed. Thus, given that ion-pair formation should already be subtracted in this analysis it is hypothesised that the 'early' stages of crystallization relate to a disordered state that slowly transforms to the crystalline gypsum perhaps via a bassanite intermediate. This is supported by the fact that after 66 minutes 50 (in Figure 9b), the broad water band at $3200 \mathrm{~cm}^{-1}$ actually decreases which can be interpreted as a decrease in the amount of disordered solids as crystallization proceeds. Similarly, in the water bend region, the broad $1640 \mathrm{~cm}^{-1}$ band completely disappears at $66 \mathrm{~min}$ to make way for a band that transforms into 55 the $1690 \mathrm{~cm}^{-1}$ gypsum/bassinite band. Thus, these results also support the non-classical crystallization mechanism.

\section{Conclusions}

What these results show is that for barite, water does not 60 appear to be playing a significant a role in crystallization when inhibitors are not present. This must be said with the caveat that the method can only probe relatively long time scales with a conventional infrared source, in the order of 2 minutes and so if the rate of water loss is sufficiently fast this may not be observed ${ }_{65}$ in these measurements. However, despite even this limitation one is able to observe the transition of the barite from a structure containing a great deal of disordered character evolving into one with more crystalline character based on peak width changes in the vibrational spectra. When inhibitor is present the disordered 70 character is longer lived and evidence for or against the role of water in stabilisation of the disordered structure was inconclusive. Method 1 suggested such a role where the presence of water decreased as structure increased, while Method 2 did not. Only further experimental work using more intense radiation 75 and much shorter sampling times will be able to shed light on the actual role of water in the stabilisation of the disordered state in the case of barite. Due to the time scales that were probed in this work, we are hoping to repeat much of this infrared work using synchrotron radiation in order to reduce the time required to 80 obtain spectra.

Experimentally, we observe gypsum to form an initial structure that is different to the final crystalline structure. The observation that these water bands increase over time may be 85 related to the movement of water from their initial disordered positions to one where the water is transforming to a crystalline solid (bassanite/gypsum). In addition, these disordered species appears quite long-lived. Thus, the presence of water may influence the stability of the disordered solid clusters. The 90 possibility arises that solution clusters might form in many different environments and this will be the subject of future work.

In this work it is seen that both systems undergo a disordered to ordered transition and this supports the non-classical view of 95 crystallization. The system will tend towards the thermodynamically stable crystal structure, the rate at which this occurs depends on the rate of removal of the entrained water molecules and/or the rate of transformation from disordered structure to crystalline product. Thus, depending on the system 100 either water removal and/or the transition to crystalline may be the slowest process. In Biomineralization, then, Nature has a choice between which pathway will better lead to the final, desired product. 


\section{Acknowledgements}

FJ would like to thank William van Bronswijk and Julian Gale and the anonymous reviewers for constructive 5 discussions/comments.

\section{Notes and references}

Curtin University of Technology, Department of Chemistry, GPO Box U1987, Perth WA Australia 6845. Fax: 6189266 4699; Tel: 6189266 10 7677; E-mail: F.Jones@curtin.edu.au

$\dagger$ Electronic Supplementary Information (ESI) available: Details of spectrum manipulation, FTIR spectrum of barium chloride at $0.25 \mathrm{mM}$, FTIR spectra of SI=100 crystallization of barite versus time, peak height and FWHM versus time, FTIR at longer times and SI=9, guassian fitting 15 of FTIR spectra of barite formed in the presence of mellitic acid versus time in water bend region. See DOI: 10.1039/b000000x/

(1) Larson, M. A.; Garside, J. Chem. Eng. Sci. 1986, 41, 1285.

(2) Erdimemir, D.; Lee, A. Y.; Myerson, A. S. Accounts of Chemical Research 2009, 42, 621.

20 (3) Ververka, V.; Söhnel, O.; Bennema, P.; Garside, J. AIChE Journal 1991, 37, 490.

(4) McLain, S. E.; Soper, A. K.; Terry, A. E.; Watts, A. Journal of Physical Chemistry B 2007, 111, 4568.

(5) Bowron, D. T.; Finney, J. L. Journal of Physical Chemistry B 25 2007, 111, 9838 .

(6) Hamad, S.; Hughes, C. E.; Catlow, C. R. A.; Harris, K. D. A. Journal of Physical Chemistry B 2008, 112, 7280.

(7) Almasy, L.; Jancso, G. Journal of Molecular Liquids 2004, 113,61 .

30 (8) Sorensen, T. J.; Sontum, P. C.; Samseth, J.; Thorsen, G.; Malthe-Sorenssen, D. Chem. Eng. Technol. 2003, 26, 307.

(9) Towler, C. S.; Taylor, L. S. Crystal Growth \& Design 2007, 7, 633.

(10) Gebauer, D.; Cölfen, H. Nano Today 2012, 6, 564.

35 (11) a) Gebauer, D.; Verch, A.; Börner, H. G.; Cölfen, H. Crystal Growth \& Design 2009, 9, 2398. b) Gebauer, D.; Völkel, A.; Cölfen, H. Science 2008, 322, 1819.

(12) Pouget, E. M.; Bomans, P. H. H.; Goos, A. C.; Fredrick, P. M.; de With, G.; Sommerdijk, N. A. J. M. Science 2009, 323, 1455.

40 (13) Piana, S.; Jones, F.; Gale, J. D. J. Am. Chem. Soc. 2006, 128, 13568.

(14) Piana, S.; Jones, F.; Gale, J. D. Crystengcomm 2007, 9, 1187.

(15) Chen, A. A.; Pappu, R. V. J. Phys. Chem. B 2007, 111, 6469.

(16) Demichelis, R.; Raitieri, P.; Gale, J. D.; Quigley, D.; Gebauer, 45 D. Nature Communications 2011, 2, 1.

(17) Anwar, J.; Boateng, P. K. J. Am. Chem. Soc. 1998, 120, 9600.

(18) Raiteri, P.; Gale, J. D. J. Am. Chem. Soc. 2010, 132, 17623.

(19) Meyer, J. L.; Eanes, E. D. Calcif. Tiss. Res. 1978, 2559.

(20) Boskey, A. L.; Posner, A. S. J. Phys. Chem, 1973, 77(19) 502313.

(21) Davey, R. J.; Blagden, N.; Potts, G. D.; Docherty, R. J. Am. Chem. Soc. 1997, 119, 1767.

(22) Almasy, L.; Jancso, G. Journal of Molecular Liquids 2004, 113,61 .

55 (23) Davey, R. J.; Blagden, N.; Righini, S.; Alison, H.; Quayle, M. J.; Fuller, S. Crystal Growth \& Design 2001, 1, 59.

(24) Sorensen, T. J.; Sontum, P. C.; Samseth, J.; Thorsen, G.; Malthe-Sorenssen, D. Chem. Eng. Technol. 2003, 26, 307.

(25) Freeman, S. R.; Jones, F.; Ogden, M. I.; Oliviera, A.; 60 Richmond, W. R. Crystal Growth \& Design 2006, 6, 2579.
(26) Miller, L. M.; Vairavamurthy, V.; Chance, M. R.; Mendelsohn, R.; Paschalis, E. P.; Betts, F.; Boskey, A. L. Biochimical et Biophysica Acta 2001, 157, 11.

(27) Nail, S. L.; White, J. L.; Hem, S. L. Journal of 65 Pharmaceutical Sciences 1976, 65, 231.

(28) Rogers, A. D.; Reeder, R. J.; Glotch, T. D. In 40th Lunar and Planetary Science Conference 2009, p paper 1202.

(29) Poduska, K. M.; Regev, L.; Boaretto, E.; Addadi, L.; Weiner, S.; Kronik, L.; Curtarolo, S. Adv. Mater. 2011, 23550.

70 (30) Parkhurst, D. L.; Appelo, C. A., "Users guide to PHREEQC (version 2) - a computer program for speciation, batch reaction, one dimensional transport, and inverse geochemical calculations" U.S Geological Survey Water-Resources Investigation Report 1999, pp 994259.

75 (31) Colville, A. A.; Staudhammer, K. American Mineralogist 1967, 52, 1877

(32) Wylde, J. J.; Allen, G. C.; Collins, I. R. Applied Spectroscopy 2001, 55, 1155 .

(33) Adler, H. H.; Kerr, P. F. American Mineralogist 1965, 50, 132.

80 (34) Schofield, P. F.; Knight, K. S.; Stretton, I. C. American Mineralogist 1996, 81, 847.

(35) Iishi, K. Physical Chemistry Minerals 1979, 4, 341.

(36) Knittle, E.; Phillips, W.; Williams, Q. Physical Chemistry Minerals 2001, 28, 630.

85 (37) Prasad, P. S. R.; Krishna Chaitanya, V.; Shiva Prasad, K.; Narayana Rao, D. American Mineralogist 2005, 90, 672.

(38) Clippe, P.; Evrard, R.; Lucas, A. A. Physical Review B 1976, 14,1715 .

(39) Johnson, B. B.; Sjöberg, S.; Persson, P. Langmuir 2004, 20, 90823.

(40) Wang, Y.-W.; Kim, Y.-Y.; Christenson, H. K.; Meldrum, F. C. Chem. Commun. 2012, 48, 504.

(41) van Driessche, A. E. S.; Benning, L. G.; Rodriguez-Blanco, J. D.; Ossorio, M.; Bots, P.; Garcia-Ruiz, J. M. Science 2012, 336, 69.

95 (42) Putnis, A.; Winkler, B.; Fernandez-Diaz, L. Mineralogical Magazine 1990, 54, 123.

(43) Chernyshova, I. V.; Hochella, M. F.; Madden, A. S. PCCP 2007, 9 .

(44) Schmidt, H.; Paschke, I.; Freyer, D.; Voigt, W. Acta 100 Crystallographica Section B 2011, 67, 467.

(45) The penetration depth is dependant on the IR wavelength as well as the refractive index of the ATR crystal, however, it varies from $0.5-2 \mu \mathrm{m}$. For small nucleating particles in the very early stages of crystallization, the particle size is expected to be in the nanometer range. 105 This is much smaller than the penetration depth and in essence means the ATR spectrum is of the entire particle (that is situated near the surface of the ATR crystal). The spectrum will therefore contain both surface and bulk bands but will none-the-less be dominated by the surface species because at these sizes the surface/bulk ratio is high. 